\title{
Design Methodology for Mass Reduction of a Mechanical Product by Extracting Minimum Structure
}

\author{
Shinichi FUKUSHIGE ${ }^{1}$, Shingo TANIYAMA², and Yasushi UMEDA ${ }^{1}$ \\ ${ }^{1}$ Department of Mechanical Engineering, Osaka University, Japan, fukushige@mech.eng.osaka-u.ac.jp \\ ${ }^{2}$ Department of Precision Engineering, The University of Tokyo
}

\begin{abstract}
:
Increasing productivity of resources is a major approach in the framework of sustainability in manufacturing. The manufacturing industry can reduce the consumption of resources and production of waste by applying more efficient design techniques. We focused on simplifying the structure of a product as the key method of design for environment. The removal of redundant components, by extracting the ideal simplified structure, decreases manufacturing costs and, at the same time, increases the resource productivity. We propose a methodology for product simplification that can be applied to general products and developed a product design support system by using information on the geometry and the connectivity of the product's components. A case study based on models of a vacuum cleaner and an inkjet printer showed the effectiveness of the methodology.
\end{abstract}

Keywords: Environmentally conscious design, Design methodology, Structure simplification

\section{Introduction}

Industries will consume fewer resources and produce less waste when more efficient designs and manufacturing techniques are used. Design techniques that reduce the amount of resources required provide benefits, such as decreased raw material consumption, reduced manufacturing costs, reduced transporting and waste disposal costs, and decreased environmental loads, to industry and the wider community.

Reducing the amount of resources used involves changes in product formulation. This paper focuses on the topic of product simplification. The majority of structure simplification has been studied as optimization techniques $[1,2,3]$. Topology and shape optimization generates the optimal mechanical structure. The structural shape is generated within a pre-defined design space, and a designer provides structural supports and loads.

However, such optimization techniques are performed under deterministic conditions and are applied to simple components, such as a frame element. Therefore, it is difficult to design a global structure of a product by using such numerical optimizations.

Conventionally, techniques to reduce the overall mass of products are developed depending on the knowledge of each product. Such techniques are limited because they can only be applied to individual products and not used as a generalized methodology.

We developed a methodology that can be applied to general products and developed a product design support system by simplifying a global structure of a product and by removing redundant components.

\section{Approach for Mass Reduction}

The method has four steps. First, "functional components”, defined as components bearing essential roles in a product, are selected. Secondly, the product model is skeletonized, and the unselected components are replaced with node points. The link elements connecting functional components and the nodes are extracted. The link represents a fixed relationship or a relative movement relationship between the components. Thirdly, redundant links are detected and removed by using topological operations so as to generate a minimum structure. The "minimum structure" is defined as the ideal simplified structure of a product. This simplification is performed until no redundant link remains. Last, a designer tries to change the layout of functional components to meet the design requirements. The minimum structure is updated interactively, and the designer creates a potential working model from the minimum structure as an example of reducing the mass of a product. We describe the process in detailed in the following section.

\subsection{Skeletonization}

First, the system imports a CAD model of a product. The model involves information about the connectivity among all components. The connectivity is classified into fixed relationships or relatively movement relationships. Next, a designer selects the functional components on the CAD model, and the system skeletonizes the model (see Figure 1). We define a functional component as a component that has an indispensable role for the overall function of the product, such as electrical controlling, power generating, and light emitting. However, it is not generally clear which components have important functions. Therefore, this selection phase depends on which components the designers want to keep or remove from the design. Most components that have functions for 
fixing, power transmission, motion guide, and shape maintenance are not selected as functional components. However, base covers and housings involved in the aesthetic design are regarded as functional components.

Secondly, the unselected components are skeletonized. Skeletonization means replacing the non-functional components with joint points. The functional components and the joint points are connected with links based on the information of physical connectivity from the CAD model.
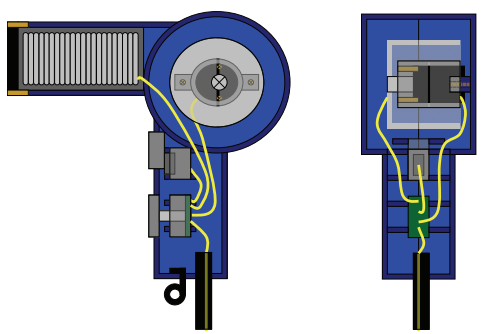

(a) Input model

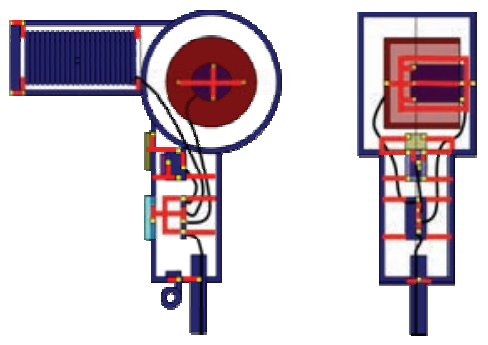

(b) Skeleton model

Figure 1: An example of skeletonization (hair dryer model)

\subsection{Extracting Minimum Structure}

After skeletonization, the skeleton model is simplified by calculating the minimum spanning trees. A minimum spanning tree of a connected graph is defined in the field of graph theory as a minimal set of edges that connects all vertices [4]. We used this algorithm to extract the minimum structure from the skeleton model.

First, a complete graph is constructed, which is a simple graph in which a link is used to connect every functional component pair with the minimal distance. This relationship is calculated using connectivity information from the skeleton. Secondly, the components are classified into some groups in which all the components have a fixed relationship within the group. The links intersecting with any other functional components are removed from the design.

Last, the minimum structure is extracted using the following procedure. The distances between all component pairs within the group are calculated initially, and all the links are labeled with the distances. As a starting point, a component is chosen and labeled, and then the following steps are performed:
(1) Select an unlabeled component nearest any labeled component and label it.

(2) Label the link connecting the selected component and its nearest component.

Steps (1) and (2) are repeated until all components are labeled. Finally, the unlabeled bones are removed, and a simplified structure remains (see Figure 2(c)). This algorithm can be shown to run in time $O(B \log C)$, where $B$ is the number of bones, and $C$ is the number of components. This algorithm is applied to all groups, and the simplified structure of an entire product is named the minimum structure.

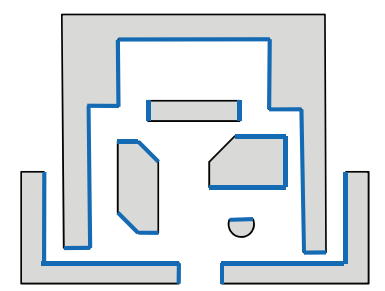

(a) two-dimensional example of functional components (The blue lines represent the interfaces that can be connected with links, and all components in this example are fixed to each other)

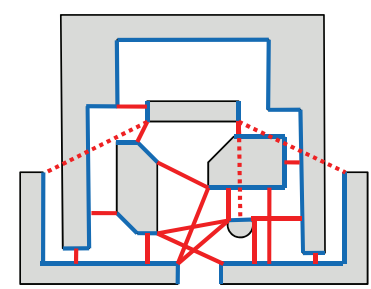

(b) A complete graph (Each link connects the components with the minimal distance. The broken lines represent unconnected links that interfere with components)

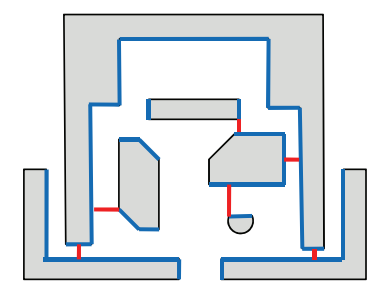

(c) Minimum structure

Figure 2: Extracting minimum structure

The minimum structure represents a structure in which the sum of all links is minimized within the fixed relationship components, and if needed, the second and third minimum structure can be proposed.

\subsection{Reconstruction of solid model}

A solid model is created from the minimum structure as a potential design. A designer can continually modify the layout of the functional components, and the minimum structure is updated interactively by the system. The 
product is constructed by the following processes:

(1) Move connected components wherever required.

(2) Change the materials to unify the components.

(3) Modify the geometry of the components or add features to fix the floating components.

The links in the minimum structure can be regarded as guides that recommend modifications to the layout and the formation of components.

\section{Case study}

The method we developed has been implemented to verify the advantages of the methodology. We used the main body of a vacuum cleaner and an inkjet printer as examples. The number of components in the vacuum cleaner model was 61 and in the printer model was 245 .

The system imported CAD models of the products, as shown in figure 3 , and connectivity information of the of the model components was generated.
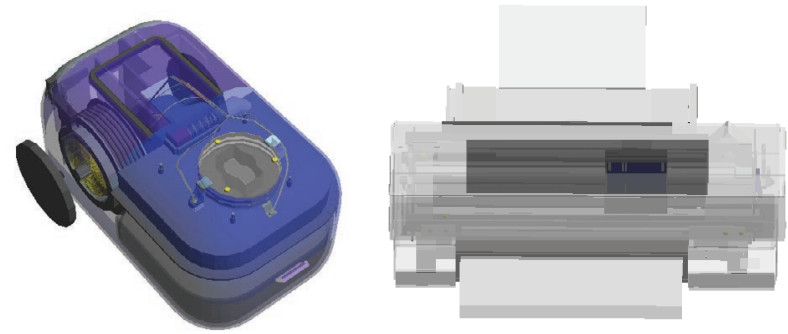

Figure 3: Geometric models of a vacuum cleaner and an inkjet printer (housing with translucency)

\subsection{Minimum Structure}

The individual functional components were selected for each model. The functional components of the vacuum cleaner model included suction, power control, energy supply, drive and aesthetic design. The motor, cover, handle, retractable cord, shaft, hose receptor, and casters were selected. Next, the models were skeletonized and simplified to create the minimum structure. Figure 4 shows the minimum structure in which the functional components are connected by a minimal set of links.
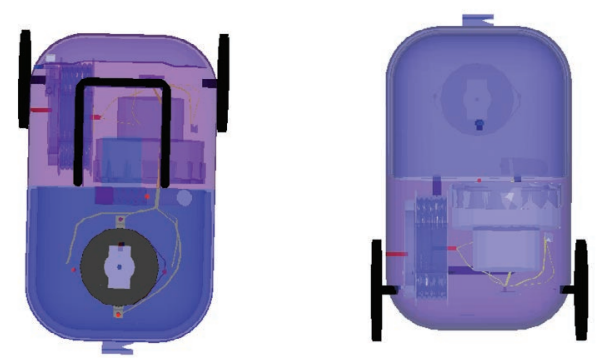

(a) Vacuum cleaner body
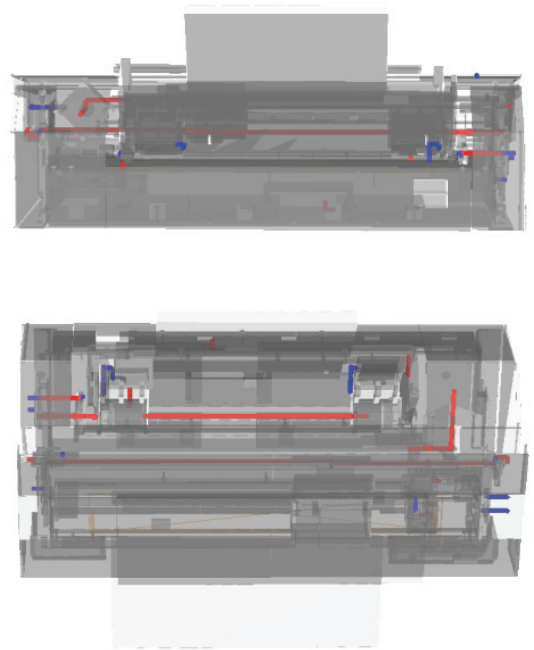

(b) Inkjet printer

Figure 4: Minimum structure

As shown in this figure, many internal components are directly connected to the housing because the housing and the base covers were selected as functional components.

\subsection{Redesign}

A designer can move the components and change the layout interactively. Figure 5 shows variations in the minimum structure based on the location of the functional components.
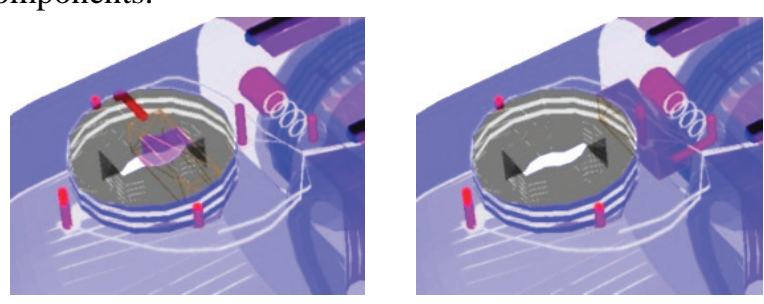

Figure 5: Transition of minimum structure (vacuum cleaner model)

Figures 6 and 7 show modified examples derived from the minimum structure. For the cleaner model, the number of components was reduced from 61 to 50 and a $6 \%$ reduction in weight was achieved. Whereas for the inkjet printer model, the number of components was reduced from 245 to 231 and a 4\% reduction in weight was achieved.

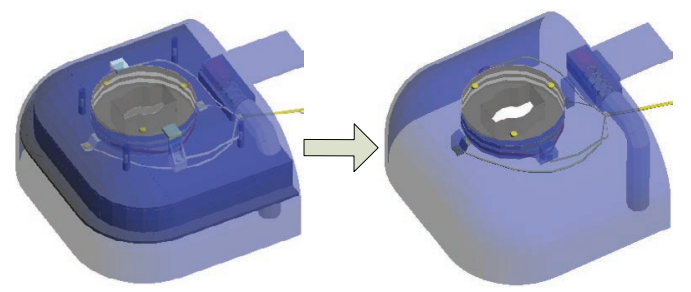

(a-1) Results of redesigning cover components 


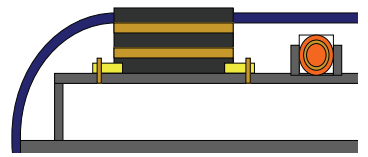

(a-2) Two-dimensional redesigning results

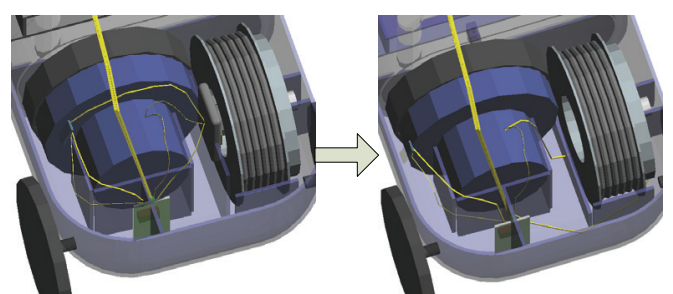

(b-1) Results of redesigning hose roll components
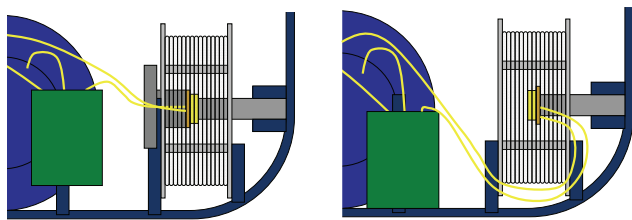

(b-2) Two-dimensional schematic diagram of redesigning results

Figure 6: Redesigned model (main body of vacuum cleaner)

As shown in Figure 6, a hose receptor has been directly fixed to the cover of the vacuum cleaner, and a base part under the cover that fixes the hose receptor has been removed.

A component in the original model, which transmits electricity from the shaft to the motor, has been moved to the right side of the shaft taking up the retractable cord. In addition, a prop component, which supports the transmission component, has also been removed.
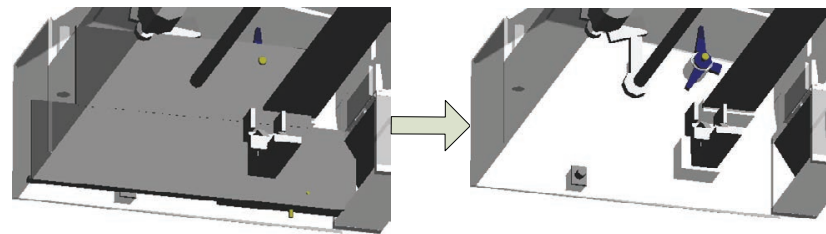

(a) Results of redesigning bearing components

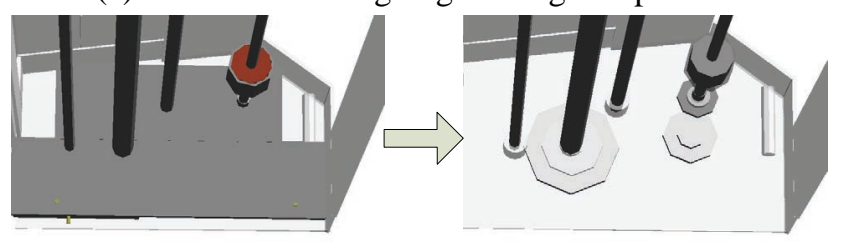

a) Results of redesigning bearing components

Figure 7: Redesigned model (inkjet printer)

The bearing plates that bear the shafts have been removed from the ink printer model, and the shafts have then been connected to the back plates, as shown in Figure 7.

\section{Discussions}

In general, mechanical structures are designed with several design requirements and have to be verified from various viewpoints, such as rigidity, deformation, and manufacturability. However, the system we developed does not consider all such design requirements. The system presents certain redesigned models derived using geometry and connectivity information from data inputted into a CAD model. It is left to the designer's discretion whether the generated model is suitable. A designer can exert their discretion on the structure of the product by considering the design intention during the component selection and redesign phases.

We cannot guarantee that all structures will be simplified using our method. However, in case the products have redundant structure, the developed method can present several solutions semiautomatically based on inputted geometrical data.

\section{Conclusion}

We developed a design methodology that reduces the mass of products. The method extracts a minimum structure as an ideal minimized formation of connectivity derived from CAD models of products. We developed the method to provide designers with a design support system that presents several potential designs that meet specific design requirements. The designer can then select a suitable design and further modify its shape and topology. A case study using models of a vacuum cleaner body and an inkjet printer showed that the design could be simplified and the number of components reduced.

Future work will involve developing techniques, such as dynamic joints, rotation, and parallel motion connectivity among components, to simplify dynamic structures.

\section{References}

[1] Bensoe, M. P. and Kikuchi, M., 1998, Generating optimal topology in structural design using a homogenization method, Computer Methods in Applied Mechanics and Engineering, Vol71-2, pp197-224.

[2] Nishigaki, H., Nishiwaki, S., Amago, T., and Kikshi, N., 2000, First order analysis for automotive body structure design, ASME DETC2000/DAC-14533.

[3] Maute, K., and Allen, M., 2004, Conceptual design of aeroelastic structures by topology optimization , Structural and Multidisciplinary Optimization, Vol.27, No. 1-2, pp.27-42.

[4] Chazelle, B., 2000, A Minimum spanning tree algorithm with inverse-Ackermann type complexity, Journal of the ACM, Vol.47, No.6, pp.1028-1047. 